\title{
Cognitive and Psychosocial Variables Predicting Gambling Behavior in a Clinical Sample
}

\author{
Tian Po S. Oei ${ }^{1,2} \cdot$ Namrata Raylu $^{1}$
}

Published online: 17 April 2015

(C) Springer Science+Business Media New York 2015

\begin{abstract}
Previous studies have mostly examined cognitive and psychosocial variables that are critical to the cognitive behavioral conceptualization of Problem Gambling (PG) in community samples. This study aimed to assess whether cognitive and psychosocial variables (gambling refusal self-efficacy, gambling cognitions, gambling urges, comorbid negative psychological states, comorbid substance use/misuse, life satisfaction and quality of life) can predict gambling behaviour in a clinical sample. One hundred and thirty-nine volunteers for a PG self-help cognitive behaviour treatment program participated. Results showed that only perceived gambling refusal self-efficacy, cognitions of inability to stop gambling, hazardous drinking behaviors, and poor quality of life related to one's environment, significantly predicted SOGS and/or CPGI scores. Significant interactions between gender and a number of variables including gambling cognitions (especially gambling expectancies), gambling refusal self-efficacy as well as quality of life related to physical or environmental health predicted gambling behaviour in this clinical sample.
\end{abstract}

Keywords Gambling $\cdot$ Cognitions $\cdot$ Urges $\cdot$ Substance $\cdot$ Mood

Cognitive behavioral models have been proposed to explain the theoretical development and maintenance of PG (Raylu and Oei 2010). Using community and student samples, psychosocial and cognitive variables that include gambling specific factors (e.g., gambling cognitions, urges, and refusal self-efficacy), gambling comorbidities, and wellbeing measures (e.g., poor life quality and satisfaction), have been cited to relate to gambling behaviors (Johansson et al. 2009; Raylu and Oei 2010). The relationships between each of these variables and gambling behaviour as well as current research limitations are further examined as below.

Tian Po S. Oei

oei@psy.uq.edu.au

1 School of Psychology, The University of Queensland, Brisbane, QLD 4072, Australia

2 James Cook University, Singapore, Singapore 
Gambling Specific Factors Gambling cognitions (misinterpreting gambling outcomes and displaying erroneous cognitions about one's winning chances and ability to predict, influence and/ or control gambling outcomes) and urges [states that motivate one to seek out gambling, which are often triggered by either internal (e.g., boredom or stress) or external (e.g., gambling cues) factors] have constantly been linked to gambling problems (Johansson et al. 2009; Raylu and Oei 2010). Pharmacotherapy and psychotherapy studies have shown that reduction in erroneous cognitions and/or urges can help to reduce gambling behaviors (Petry 2009; Oei et al. 2010). In addition, gambling cognitions and urges have also been cited to significantly predict gambling behaviors among community samples and be linked to gambling relapse following a period of abstinence (Hodgins and el-Guebaly 2004; Oei et al. 2008a; Raylu and Oei 2004a, b).

Evidence from these studies suggests a direct relationship between these two gambling factors and gambling behaviors. However, most of these findings are based on community samples. Thus, more robust evidence of the effects of gambling cognitions and urges especially in clinical samples are needed.

Gambling refusal self-efficacy refers to one's belief in resisting gambling in a particular circumstance (Casey et al. 2008). Gambling refusal self-efficacy has been found to correlate negatively with gambling and PG behaviors (Hodgins et al. 2004; Casey et al. 2008). However, most of these studies were based on community or student samples (i.e., Casey et al. 2008) and pathological gamblers who had recently stopped gambling (Hodgins et al. 2004). While cognitive behavioral studies have demonstrated significant improvements in gambling refusal self-efficacy among treatment completers, selfefficacy was assessed using two or three items or instruments not properly validated in these studies (Casey et al. 2008).

Although gambling refusal self-efficacy has been found to be associated with gambling and PG behaviors, methodological shortcomings render the need for further replication of the suggested relationship between gambling self-efficacy and gambling behavior, especially in clinical samples.

Co-Morbid Problems Several studies have shown higher levels of co-morbid problems including stress, anxiety, depression and substance-related problems (Ste-Marie et al. 2006; Westphal and Johnson 2007) among problem and at-risk gamblers as compared to social, infrequent, and/or non-PGs. Additionally, comorbidity in psychiatric disorders and negative mood states among PGs is related with greater severity of gambling problems and higher likelihood of gambling relapse following a period of abstinence (e.g., Ste-Marie et al. 2006; Westphal and Johnson 2007; Rush et al. 2008). Similarly, negative psychological states and substance misuse also predict gambling behaviour among community samples (Oei et al. 2008a, b). These studies highlighted the direct impact of comorbid problems on problem gambling manifestations among PGs.

While these findings suggest that negative psychological states and substance misuse predict gambling behavior among PGs and community individuals, and were also able to predict severity of gambling behavior among PGs, evidence on this relationship remains limited and could benefit from replication and examination with other gambling correlates such as cognitive variables as discussed above.

Well-Being Correlates A handful of studies have found lower quality of life among PGs compared to non-PGs (Carlbring and Smit 2008; Sander and Peters 2009). However, these studies reported quality of life as outcomes of problem gambling. To the authors' knowledge, no studies to date have assessed the predictive value of quality of life on gambling behavior. 
On the other hand, only a small number of studies have assessed life satisfaction among PGs, and these studies have reported conflicting results. For instance, Winslow (2002) found no significant difference between non-gamblers, PGs and non-PGs on life satisfaction among an elderly community sample. However, Wong et al. (2008) found a negative relationship between life satisfaction and PG among 198 university students in Macao. Lai (2006) also found that a high level of life satisfaction negatively predicted gambling behavior among a community sample of elderly Chinese residing in Canada. Currently, there are limited evidence (mostly using community samples) citing the predictive effects of quality of life on gambling behavior, and inconsistent results representing the relationship between life satisfaction and gambling behavior. The effects of quality of life and life satisfaction on gambling behavior especially in clinical samples deserve further attention.

\section{Study Aims and Hypotheses}

In all, gambling refusal self-efficacy, gambling cognitions, gambling urges, comorbid negative psychological states, comorbid substance use/misuse, life satisfaction and quality of life have been shown to be important in the development and maintenance of gambling problems. However, these variables have either been studied independently of each other or are tested using community samples. Therefore, the present study aimed to compensate for these limitations by assessing whether these seven variables are significantly related to gambling behaviour in the clinical population.

In the gambling literature, gambling behaviour has been mainly assessed by the South Oaks Gambling Scale (SOGS; Lesieur and Blume 1987) and Canadian Problem Gambling Index (CPGI; Ferris and Wynne 2001). Although both of these measures are reported to have adequate psychometric properties, studies comparing both instruments have favored the use of the CPGI due to its better psychometric properties; its reduced likelihood of overestimating PG rates, having more variable items and being better at differentiating regular and problem gamblers (Neal et al. 2005). Considering the variances in these two instruments, it is possible that the seven variables will predict SOGS and CPGI scores differently. Thus, this study will also explore whether the seven variables can predict both the SOGS and CPGI scores equally well in a clinical sample.

\section{Method}

\section{Participants}

Participants $(n=139)$ were volunteers who took part in a self-help cognitive behaviour treatment program for gambling problems. Overall, 144 participants (of the 175 recruited) completed pre-treatment questionnaires. However, this study reported on 139 of these participants, as pre-treatment data of five of participants were unavailable when this study was written up.

The first 101 participants were recruited in Brisbane by project staff at the University of Queensland, with the use of (1) a central website that included the completion of an online participation form and (2) media announcements on radio, newspaper articles and advertisements, and flyers/posters distributed across various universities and mental health providers in 
Queensland. The remaining 74 participants were recruited via the Gambling Hotline in Melbourne, Victoria, under the management of Turning Point Drug and Alcohol Services.

The inclusion criteria included: (a) willingness to be randomly assigned to one of the treatment conditions or a waitlist condition for 6 weeks prior to commencing treatment; and (b) over 18 years old. Exclusion criteria included: (a) suicidal ideation; (b) involvement in legal proceedings requiring documentation of treatment; (c) already undergoing specific gambling treatment; (d) receiving treatment for severe psychiatric co-morbidity, schizophrenia, and mania; and (e) inadequate proficiency in English.

Of the 139 participants, $53.3 \%$ were males and $46.7 \%$ were females. Mean age of the participants was $42.65(S D=15.15$; age range $=16-78$ years $)$. Approximately $35.8 \%$ reported their annual income to be less than $\$ 20,000,18.2 \%$ reported it being $\$ 20,000-\$ 40,000$, $24.8 \%$ reported it being $\$ 40,000-\$ 60,000$, and $21.2 \%$ reported earning over $\$ 60,000$ per annum. Most participants reported their highest level of education as secondary $(61.7 \%)$. Others reported their highest level of education as certificate/diploma (20.5\%), bachelors or higher degree (14.7\%) or primary (3.1\%). Approximately $30.9 \%$ were single, $44.9 \%$ were married/in a relationship (including defacto relationship), $20.5 \%$ were divorced or separated, and $3.7 \%$ reported being widowed. $47.1 \%$ reported being employed, and others reported being employed either part-time (18.2\%), being a full-time student $(5.8 \%)$, being retired (7.2\%), looking for work $(5.8 \%)$, doing home-duties $(2.9 \%)$ or receiving unemployment or sickness/disability benefit $(13.0 \%)$. Most participants $(76.4 \%)$ identified themselves as Caucasians and were born in Australia (71.9\%). There were no significant differences in any of the demographic factors between the two recruitment sites. Thus, the data was combined for analyses.

\section{Measures}

All participants were asked questions on gender, income, education, age, marital status, employment, and ethnic background. Measures were used in this study are described below.

SOGS The SOGS is a 20-item self-report scale that assesses gambling problems. Participants indicated whether each statement applied to them over the past month and were classified as having PG with a SOGS total (T) score of five or higher. The SOGS has shown to have good internal consistency $(\alpha=.86)$ and correlated well with other measures of gambling problem such as CPGI (see below).

CPGI The CPGI measured pathological gambling risk using only nine out of 31 items in the questionnaire. Participants indicated the extent to which each statement applied to them over the past 12 months based on a four-point Likert scale $(0=$ Never, $1=$ Sometimes, $2=$ Most of the time, and 3=Almost always), and were identified as PGs if their CPGI-T score was between 8 and 27 points. The CPGI has shown good internal consistency $(\alpha=.84)$ and good criterionrelated validity with other measures of gambling problem e.g., SOGS $(r=.83)$.

Gambling Related Cognitions Scale (GRCS; Raylu and Oei 2004a) The GRCS is a 23-item questionnaire for assessment of a range of gambling cognitions. The five subscales include: (a) illusion of control (GRCS-IC), (b) predictive control (GRCS-PC), (c) interpretative bias (GRCS-IB), (d) gambling related expectancies (GRCS-GE), and (e) perceived inability to stop gambling (GRCS-IS). Participants used a seven-point Likert scale, ranging from one 
(strongly disagree) to seven (strongly agree), to indicate their extent of agreement with the value expressed in each statement. The scale has high internal consistency $(\alpha=.93)$. The total score could classify participants into two groups (SOGS $=0$ and $\mathrm{SOGS} \geq 4$ ), suggesting good criterion related validity $\left(\Lambda=.88, \chi^{2}=185, p<.001\right)$.

Gambling Urge Scale (GUS; Raylu and Oei 2004b) The GUS is a six-item questionnaire screening for gambling-related urges. Participants used a seven-point semantic differential scale to indicate their extent of agreement or disagreement with each statement based on their thoughts and feelings during completion of the GUS. The scale has high internal consistency $(\alpha=.81)$. The GUS-T score could classify participants into two groups ( $\mathrm{SOGS}=0$ and $\mathrm{SOGS} \geq 4)$, suggesting good criterion related validity $\left(\Lambda=.83, \chi^{2}=\right.$ $130, p<.001)$.

The Depression Anxiety Stress Scale-21 (DASS; Lovibond and Lovibond 1995) The DASS-21 is a 21-item version of the DASS-42 self-report questionnaire that assesses levels of depression, anxiety, and stress. Participants used a four-point Likert scale ( $0=$ Did not apply to me at all to 4=Applied to me very much, or most of the time) to indicate the extent that each statement applied to them over the past week. The DASS-21 has high internal consistency (i.e., $\alpha=.94, .87$, and .91 for the depression, anxiety, and stress subscales, respectively; Antony et al. $1998)$ and has shown to have high concurrent validity $(r=.84)$.

Gambling Refusal Self-Efficacy Questionnaire (GRSEQ; Casey et al. 2008) is a 31-item questionnaire that assesses the respondent's level of confidence in gambling refusal using a 11point Likert scale, ranging from zero (No confidence, cannot refuse) to 100 (Extreme confidence, certain can refuse) in four circumstances/subscales, i.e., (a) under influence of drugs (GRSEQ-DR); (b) when experiencing negative emotions (GRSEQ-NE); (c) when experiencing positive emotions (GRSEQ-PE); and (d) when in high-risk situations such as seeing others gamble (GRSEQ-ST). The total and subscale scores have shown to have high internal consistency ( $\alpha$ from .92 to .98 ) as well as good criterion-related validity with SOGS (significant negative $r$ ranging from .56 to .83 ).

Satisfaction with Life Scale (SWLS - Diener et al. 1985) The SWLS is a five-item questionnaire measuring global life satisfaction. Participants used a seven-point Likert scale, ranging from one (strongly disagree) to seven (strongly agree), to indicate their extent of agreement with the value expressed in each statement. The questionnaire has found to have good internal consistency $(\alpha=.82)$ and good convergent validity with other measures of subjective well being ( $r$ was found to be .50 or higher).

Alcohol Use Disorders Identification Test (AUDIT - Saunders et al. 1993) The AUDIT is a 10-item three-factor questionnaire. The factors assess hazardous alcohol use (e.g., high frequency and quantity of drinking); dependence symptoms (e.g., drinking alcohol in the mornings); and harmful alcohol use (e.g., experiencing blackouts). Participants responded on a scale ranging from zero (e.g., never drinks) to four (e.g., four times a week), with a score of eight or more for males and seven for females indicating strong likelihood of hazardous or harmful alcohol consumption and a score of more than 20 for both gender suggesting alcohol dependence. The AUDIT has been found to have high intrascale reliability among drinkers $(\alpha=.93)$. It has the ability to differentiate between those with hazardous/ harmful alcohol use and those without. 


\section{World Health Organisation Quality of Life - Brief (WHOQOL-Bref, the WHOQOL}

Group 1998) The WHOQOL-bref is a 26-item version of the WHOQOL-100 questionnaire that assesses changes in the quality of life in the last 4 weeks. Four factors of the questionnaire (i.e., physical health, psychological well being, social relationships and the environment) measure how participants feel about their quality of life based on a Likert scale, ranging from one (e.g., very poor, very dissatisfied, or not at all) to five (e.g., very good, very satisfied, extreme amount, or completely). The WHOQOL-bref showed good internal consistency for the domains ranging from .66 to .84 . The questionnaire also showed good discriminant validity by discriminating between those that were ill and those that were not.

With the current sample, Cronbach's coefficient alphas of all the total scores were above .80 (ranged from .85 to .96), except SOGS-T score $(\alpha=.77)$. Cronbach's coefficient alphas of all the subscale scores were above .70 (ranged from .71 to .96), except GRCS-GE ( $\alpha=.67$ ).

\section{Procedures}

Participants that volunteered for the study were assigned to Self Help CBT (i.e., completing a self-help CBT manual; Oei et al. 2008b), Telephone Counselling or Telephone Counselling plus Self Help CBT conditions. In the Telephone Counselling condition, participants received six sessions of telephone counselling at scheduled times during the week. The telephone counselling sessions followed the format of the self-help CBT manual.

Volunteers who agreed to participate in the study received a set of questionnaires (in the same order) at pre-treatment, post treatment and at follow-ups via mail and returned the completed questionnaires in stamped, self-addressed envelopes. Only the pre-treatment data was used for this study.

\section{Data Analyses}

Data were explored using Statistical Package for the Social Sciences 15. First, the relationships between the cognitive and psychosocial variables and gambling behaviour were assessed via zero-order correlations. Second, the extent to which the seven cognitive and psychosocial variables predicted gambling behaviour (i.e., SOGS-T score as well as CPGI-T score) was assessed via series of hierarchical multiple regression (HMR) analyses. Considering significant gender differences found in gambling behaviour and gambling correlates (Raylu and Oei 2010), gender was controlled for in all regression analyses.

\section{Results}

\section{Correlations Between Cognitive and Psychosocial Variables \& Gambling Behaviour}

Positive significant correlations were found between GRCS-T and subscale scores and SOGS-T score. Significant positive correlations were also found between CPGI-T scores and the GRCS-T score. However, only two of the GRCS subscale scores (i.e., GRCS-IC and GRCS-IB) significantly (and positively) correlated with CPGI-T scores. Significant positive correlations were also found between the two dependent variables (CPGI-T and SOGS-T scores) and other assessed variables (including GUS-T, DASS-T and DASS subscale scores). 
Significant negative correlations were found between the two dependent variables (CPGI-T and SOGS scores) and the GRSEQ-T and subscale scores (except between CPGI-T and GRSEQ-DR subscale score). Significant negative correlations were also found between the two dependent variables (CPGI-T and SOGS-T scores) and other assessed variables (including SWLS-T score and both WHOQOL-T and subscale scores).

The relationship between AUDIT-T and subscale scores and gambling behaviour (both CPGI-T and SOGS-T scores) were non-significant. Correlations between the assessed cognitive and psychosocial variables and gambling behaviour (CPGI-T and SOGS-T scores) are displayed in Tables 1, 2 and 3.

\section{Extent to Which Cognitive and Psychosocial Variables can Predict Gambling Behaviour}

\section{Prediction of SOGS Scores}

The first HMR analysis was conducted using the SOGS-T score as the dependent variable and the total scores of each of the seven cognitive and psychosocial variables as the independent variables. Therefore, (i) gender (first step), (ii) GRCS-T, GUS-T, GRSEQ-T, DASS-T, AUDITT, SWLS-T and WHOQOL-T scores (second step), and (iii) two-way interactions between gender and the total scores of the seven measures (third step) were used as predictor variables in the HMR analysis. Results (displayed in Table 4) showed that $32 \%$ of the variance (adjusted $\left.R^{2}=21 \%\right)$ in SOGS scores was accounted for by the predictors $(R=.57, p<.01)$. Only the GRSEQ-T score (accounted for $7 \%$ of the variance) contributed significantly to the prediction of the SOGS scores.

The second HMR analysis was conducted using the SOGS-T score as the dependent variable and the subscale scores of the seven measures as the independent variables. Thus, (i) gender (first step), (ii) GRCS-GE, GRCS-IC, GRCS-PC, GRCS-IS and GRCS-IB, GRSE Q-ST, GRSEQ-DR, GRSEQ-PE, GRSEQ-NE, GUS-T, DASS-D, DASS-A, DASS-S, AUDIT-HZ, AUDIT-DS, AUDIT-HM, SWLS-T, WHOQOL-PH, WHOQOL-PW, WHOQOL-SR and WHOQOL-BE (second step), and (iii) two-way interactions between gender and the subscale scores of the seven measures (third step) were used as predictor variables in the HMR analysis. Results (refer to Table 5) showed that $56 \%$ of the variance (adjusted $\left.R^{2}=30 \%\right)$ in SOGS scores was accounted for by the predictors $(R=.75, p<.01)$. Only the WHOQOL-E subscale score (accounted for $10 \%$ of the variance) contributed significantly to the prediction of the SOGS scores.

\section{Prediction of CPGI Scores}

The third HMR analysis was the same as the first HMR, except the dependent variable was the CPGI-T rather than SOGS-T. Results showed that $37 \%$ of the variance (adjusted $R^{2}=27 \%$ ) in CPGI-T scores was accounted for by the predictors $(R=.61, p<.001)$. Only the gender $\mathrm{x}$ GRCS-T interaction (accounted for $11 \%$ of the variance) contributed significantly to the prediction of the CPGI-T scores. The results are displayed in Table 4.

The fourth HMR analysis was the same as the second HMR, except the dependent variable was the CPGI-T rather than SOGS-T. Results showed that $64 \%$ of the variance (adjusted $R^{2}=$ $43 \%)$ in CPGI scores was accounted for by the predictors $(R=.80, p<.001)$. Only GRCS-IS, AUDIT-HZ, gender x GRCS-GE interaction, gender x GRSEQ-ST, gender $x$ WHOQOL-PH 


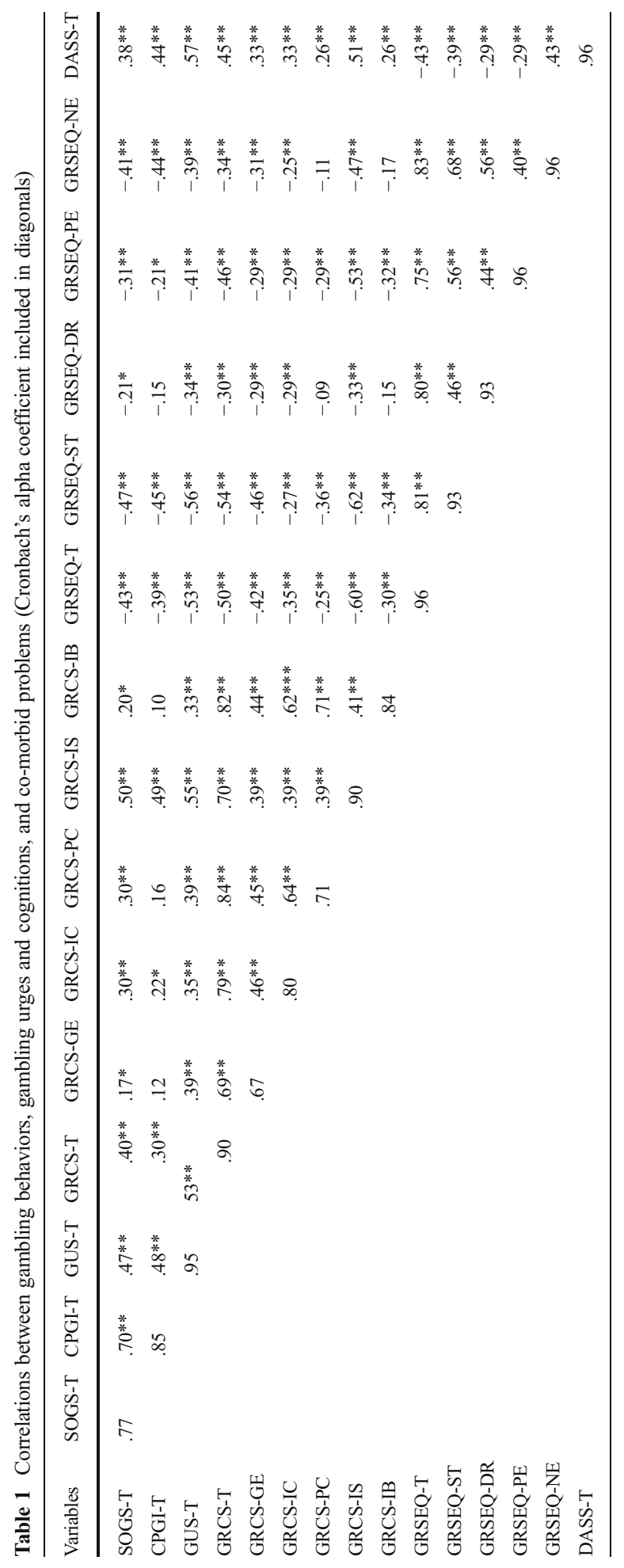




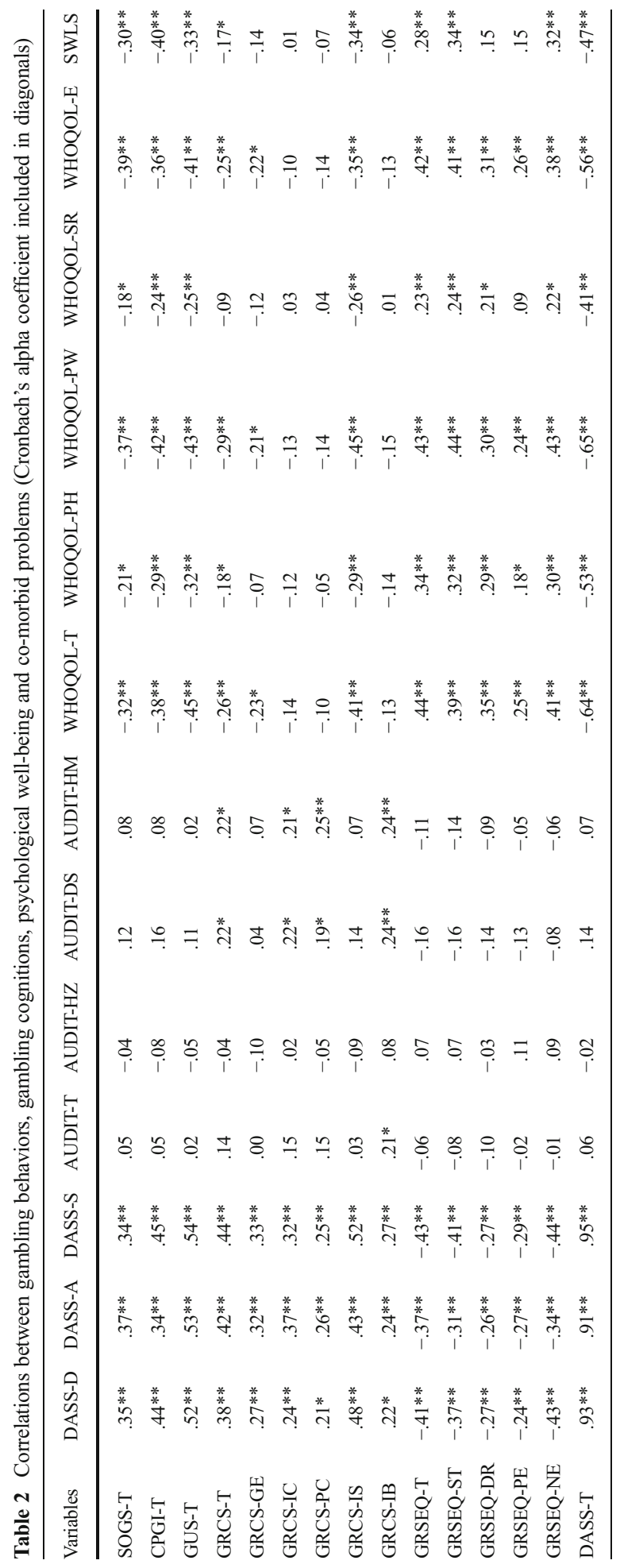




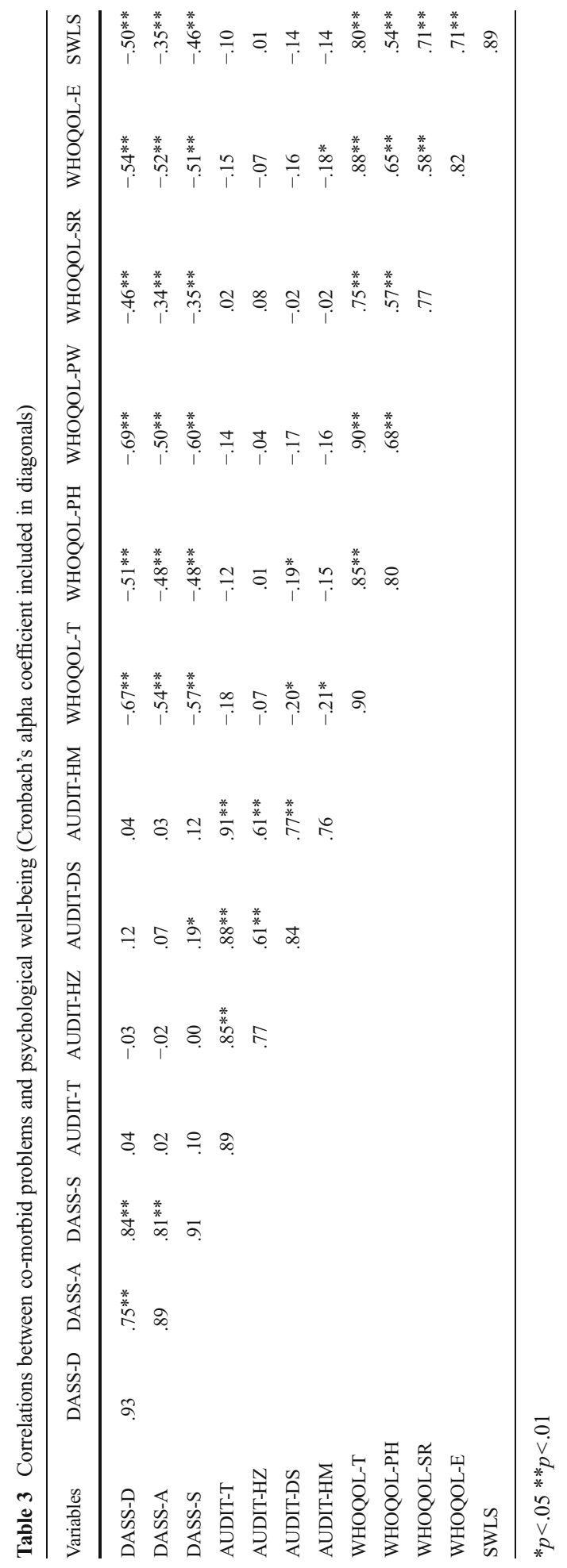


Table 4 Hierarchical multiple regression of the interactions between gender and the assessed cognitive and psychosocial variables (total scores) predicting gambling behaviour

$* p<.05 * * p<.01$

\begin{tabular}{|c|c|c|c|}
\hline Step & Independent variables & $\begin{array}{l}\text { Beta when SOGS } \\
\text { score is the dependent } \\
\text { variable }\end{array}$ & $\begin{array}{l}\text { Beta when CPGI } \\
\text { score is the dependent } \\
\text { variable }\end{array}$ \\
\hline 1 & Gender & .01 & .08 \\
\hline \multirow[t]{7}{*}{2} & GUS-T & .15 & .25 \\
\hline & GRCS-T & .14 & -.05 \\
\hline & GRSEQ-T & $-.26^{*}$ & -.12 \\
\hline & DASS-T & .09 & .23 \\
\hline & AUDIT-T & .02 & .02 \\
\hline & WHOQOL-T & .00 & .05 \\
\hline & SWLS-T & -.09 & -.17 \\
\hline \multirow[t]{7}{*}{3} & $\begin{array}{l}\text { Gender x GUS-T } \\
\text { interaction }\end{array}$ & .08 & -.12 \\
\hline & $\begin{array}{l}\text { Gender x GRCS-T } \\
\text { interaction }\end{array}$ & .08 & $.33^{*}$ \\
\hline & $\begin{array}{l}\text { Gender x GRSEQ-T } \\
\text { interaction }\end{array}$ & .08 & .12 \\
\hline & $\begin{array}{l}\text { Gender x DASS-T } \\
\text { interaction }\end{array}$ & -.03 & -.18 \\
\hline & $\begin{array}{l}\text { Gender x AUDIT-T } \\
\text { interaction }\end{array}$ & .00 & -.08 \\
\hline & $\begin{array}{l}\text { Gender x WHOQOL-T } \\
\text { interaction }\end{array}$ & -.06 & -.25 \\
\hline & Gender x SWLS-T & .04 & .17 \\
\hline
\end{tabular}

and WHOQOL-E contributed significantly to the prediction of the CPGI scores. The proportion of variance in CPGI scores were accounted for by $11,7,6,20,15$, and $8 \%$ of the variance in GRCS-IS, AUDIT-HZ, gender x GRCS-GE interaction, gender x GRSEQ-ST, gender $\mathrm{x}$ WHOQOL-PH and WHOQOL-E respectively. The results are displayed in Table 5.

\section{Discussion}

The study found that, unlike what was expected, not all cognitive and psychosocial variables could predict gambling behaviour in this clinical sample. Only GRSEQ-ST, GRCS-IS, AUDIT-HZ and WHOQOL-E could significantly predict SOGS-T and/or CPGI-T. The findings are discussed in detail below.

Gambling Cognitions Raylu and Oei (2004a) had reported that four of the five GRCS subscales could predict gambling behaviour among their community sample. However, only perceived inability to stop/control gambling could predict gambling behaviour (i.e., CPGI-T) in this clinical sample. The inability to stop gambling is a characteristic that distinguishes nonPGs from PGs. Thus, findings from this study supported the predictive effects of perceived inability to stop gambling on gambling behavior, which are applicable only to more regular gamblers and those with more severe gambling problems. PGs often have many unsuccessful attempts to stop gambling (Raylu and Oei 2010). This can reinforce cognitions relating to their 
Table 5 Hierarchical multiple regression of the interactions between gender and the assessed cognitive and psychosocial variables (subscale scores) predicting gambling behaviour

\begin{tabular}{|c|c|c|c|}
\hline Step & Independent variables & $\begin{array}{l}\text { Beta when SOGS score } \\
\text { is the dependent variable }\end{array}$ & $\begin{array}{l}\text { Beta when CPGI } \\
\text { score is the dependent } \\
\text { variable }\end{array}$ \\
\hline 1 & Gender & .01 & .05 \\
\hline \multirow[t]{21}{*}{2} & GUS-T & .01 & .22 \\
\hline & GRCS-GE & -.08 & -.14 \\
\hline & GRCS-IC & .17 & .15 \\
\hline & GRCS-PC & .06 & .11 \\
\hline & GRCS-IS & .23 & $.34 * *$ \\
\hline & GRCS-IB & -.10 & -.27 \\
\hline & GRSEQ-ST & -.16 & -.04 \\
\hline & GRSEQ-DR & .04 & .15 \\
\hline & GRSEQ-PE & .01 & .17 \\
\hline & GRSEQ-NE & -.16 & -.20 \\
\hline & DASS-D & .11 & .06 \\
\hline & DASS-A & .10 & -.02 \\
\hline & DASS-S & -.20 & -.02 \\
\hline & AUDIT-HZ & -.13 & $-.27^{*}$ \\
\hline & AUDIT-DS & .09 & .24 \\
\hline & AUDIT-HM & .04 & .00 \\
\hline & WHOQOL-PH & .15 & -.10 \\
\hline & WHOQOL-PW & -.11 & -.01 \\
\hline & WHOQOL-SR & .17 & .12 \\
\hline & WHOQOL-E & $-.31^{*}$ & -.07 \\
\hline & SWLS-T & -.10 & -.18 \\
\hline \multirow[t]{21}{*}{3} & Gender $x$ GUS-T interaction & .11 & -.07 \\
\hline & Gender x GRCS-GE interaction & .19 & $.24 *$ \\
\hline & Gender x GRCS-IC interaction & -.07 & -.07 \\
\hline & Gender x GRCS-PC interaction & .12 & .25 \\
\hline & Gender x GRCS-IS interaction & .24 & .20 \\
\hline & Gender $x$ GRCS-IB interaction & -.19 & -.05 \\
\hline & Gender x GRSEQ-ST interaction & .28 & $.45^{* *}$ \\
\hline & Gender x GRSEQ-DR interaction & -.02 & .02 \\
\hline & Gender x GRSEQ-PE interaction & -.21 & -.18 \\
\hline & Gender x GRSEQ-NE interaction & .08 & -.04 \\
\hline & Gender x DASS-D interaction & .29 & .05 \\
\hline & Gender $x$ DASS-A interaction & -.32 & -.19 \\
\hline & Gender x DASS-S interaction & -.10 & -.13 \\
\hline & Gender $\mathrm{x}$ AUDIT-HZ interaction & .15 & .00 \\
\hline & Gender x AUDIT-DS interaction & -.15 & -.01 \\
\hline & Gender x AUDIT-HM interaction & .09 & .04 \\
\hline & Gender $x$ WHOQOL-PH interaction & -.18 & $-.39 *$ \\
\hline & Gender $\mathrm{x}$ WHOQOL-PW interaction & .08 & -.00 \\
\hline & Gender $x$ WHOQOL-SR interaction & -.11 & -.11 \\
\hline & Gender x WHOQOL-E interaction & .18 & $.28^{*}$ \\
\hline & Gender $x$ SWLS-T interaction & -.10 & .02 \\
\hline
\end{tabular}

${ }^{*} p<.05 * * p<.01$ 
inability to stop or control their gambling behaviors. Consequently, this category of cognitions among PGs becomes more focused and automatic.

The study also found that an interaction between gambling cognitions (especially cognitions related to individuals' perceived expectations about the effects of gambling) and gender could also predict gambling behaviour. Post-hoc results showed that no significant gender differences were found in gambling behaviour (CPGI-T) among those with low gambling expectancies. However, females with high gambling expectancies had higher gambling behaviour (CPGI-T) than males with high gambling expectancies. The GRCS subscale that assesses cognitions related to individuals' perceived expectations about the effects of gambling only assesses positive expectancies. Thus, this interaction effect supports findings from Toneatto et al.'s (2002) study, which reported female PGs endorsing more positive expectancies than male PGs. On the other hand, male and female non-PGs showed similar levels of positive expectancies (Toneatto et al. 2002).

Gambling Refusal Self-Efficacy Gambling refusal self-efficacy could also predict gambling behaviour (SOGS-T) in this clinical sample. Gambling refusal self-efficacy, which relates to one's perceived ability to resist gambling in certain situations, is related to the gambling cognitions (i.e., perceived inability to stop gambling).

The study also found that an interaction between gender and gambling refusal self-efficacy when in high-risk situations or when experiencing high-risk thoughts could also predict gambling behaviour among this clinical sample. Post-hoc results showed that higher gambling refusal self-efficacy was related with less frequent gambling (CPGI-T) for males, but not for females. This implies that the buffering effects of gambling refusal self-efficacy on gambling behavior may be more relevant for male PGs. Further validation of the observed relationship between gambling refusal self-efficacy and gambling behaviour using different samples is needed to affirm this link.

Gambling Urges The study did not show that gambling urges predicted gambling behaviour (both CPGI-T or SOGS-T), despite previous studies showing positive relationships between gambling urges and gambling behaviors in both community and clinical samples (e.g., Oei et al. 2008a, b). While this was unexpected, it is possible that gambling urges were highly prevalent and more evenly distributed among the clinical sample test in this study, whereas a wider range of gambling urges and behaviors can be experienced by community individuals who may or may not be PGs. This may at least partially explain why gambling urges can predict gambling behaviour among community samples but not for the sample in this study.

Comorbid Problems Negative psychological states did not predict gambling behaviour (both SOGS-T and CPGI-T). However, hazardous alcohol use (e.g., high frequency and quantity of drinking) could significantly predict gambling behaviour (CPGI-T) among this sample. This finding supported results of Rush et al.'s (2008) study, which found that in a household survey of 36,885 participants, the prevalence of PG was influenced by the severity of comorbid substance use disorders but not comorbid mood and anxiety disorders.

Life Satisfaction Life satisfaction did not predict gambling behaviour among the clinical sample. This was similar to what was found with Wong et al.'s (2008) study with university students but contradictory to Lai's (2006) study with the elderly. It is possible that satisfaction with life is predictive of gambling behavior in specific populations (e.g., the elderly; Lai 2006). 
Further research is required to assess the relationship between life satisfaction and gambling behaviour among different samples to assess such hypothesis.

Quality of Life Only quality of life in relation to environmental health could predict gambling behaviour (SOGS-T). Furthermore, results showed that for males, the higher the quality of life in relation to environmental health, the lower the CPGI-T; for females, this relationship was not significant. On the other hand, for females, higher quality of life in relation to physical health is related to lower CPGI-T scores. For males, this relationship was not significant. Possibly, quality of life in relation to environmental health is more important in predicting gambling behaviour among males, whereas quality of life in relation to physical health is vital in predicting gambling behaviour among females. This supported findings of Crisp et al. (2000), which reported that male treatment-seeking PGs reported concerns with external problems (e.g., finances), whereas female treatment-seeking PGs reported concerns with internal problems (e.g., physical health).

Measures of Gambling Behavior This study found that the CPGI (37\% total and $63 \%$ subscales) was better than the SOGS (32\% total and $56 \%$ subscales) in measuring the predictive ability of cognitive and psychosocial variables on gambling behaviour. This is not surprising considering that a number of comparison studies that have favored the use of CPGI over SOGS, highlighting that the CPGI has better psychometric properties, items and ability to estimate prevalence rates than the SOGS (Neal et al. 2005). This study also found that the reliability (alpha coefficient) of the CPGI $(\alpha=.85)$ was superior to the SOGS $(\alpha=.77)$. Thus, this study further supports the utility of CPGI over the SOGS.

In contrast to study expectations, many variables were not predictive of gambling behaviour in the clinical sample tested. There are a number of possible reasons. First, it is possible that this was due to a ceiling or floor effect. That is, the scores for some of these measures were either generally on the high end of the score range-ceiling effect (e.g., CPGI-T and some of the GRCS subscale scores) or on the low end of the score range-floor effect (e.g., DASS-A and GRCS-IC subscale scores as well as AUDIT-T and GRSEQ-T and some of the subscale scores). Second, variables such as gambling cognitions are so well-rehearsed and thus, have become automatic among PGs in clinical samples (and thus, are not easily detected by measuring instruments) as compared with the non-PGs. It is to be noted that such explanations are preliminary and further replication of findings in this study is recommended for a sharper understanding of the relationships explicated in this study.

Study Implications Findings of this study highlighted the importance of targeting gambling cognitions, especially in their perceived inability to stop gambling and belief that they could resist gambling high-risk situations or thought during treatment. This is important as PGs' confidence in their ability to refuse or stop gambling can impact on the extent to which they try to control/stop gambling or relapse into gambling (Raylu and Oei 2004a). Findings of this study also support conclusions drawn from Rush et al. (2008) study that treatment may also need to target substance use disorders among those presenting with comorbid gambling and substance use problems. Finally, considering the gender differences found in this study, there might be a need to appreciate gender differences in treatment needs of PGs. 
Study Limitations Since all participants in this study were volunteers, the sample would have mainly consisted of self-motivated individuals. Future studies need to replicate the findings with PGs in other clinical populations of (e.g., those in inpatient treatment programs or PGs coerced into treatment).

In summary, the study showed the importance of gambling cognitions, gambling related self-efficacy, quality of life and comorbid variables such as alcohol use/misuse in predicting gambling behaviors among a clinical sample of PGs.

Acknowledgments We would sincerely like to thank Shana Grozdanovic and Jasmine Loo for their assistance with this study. This research project was supported financially by the Queensland Office of Gaming Regulation under the Responsible Gambling Research Grants Program. The findings of the study were submitted to the Queensland Office of Gaming Regulation as a condition of the grant. Parts of the findings of this study were presented at the Asian Psychological Association, Darwin, July 2010.

Conflict of Interest On behalf of all authors, the corresponding author states that there is no conflict of interest.

Disclaimer This study presents the findings of independent research. These findings and the associated argument and interpretation derive from the project as conducted, and do not necessarily represent or reflect the views of the Queensland Government or the Queensland Office of Gaming Regulation.

\section{References}

Antony, M. A., Bieling, P. J., Cox, B. J., Murray, W. E., \& Swinson, R. P. (1998). Psychometric properties of 42 item and 21-item version of the Depression, Anxiety and Stress Scale in clinical groups and a community sample. Psychological Assessment, 10, 176-181.

Carlbring, P., \& Smit, F. (2008). Randomized trial of internet-delivered self-help with telephone support for pathological gamblers. Journal of Consulting \& Clinical Psychology, 76, 1090-1094.

Casey, L. M., Oei, T. P. S., Melville, K. M., Bourke, E., \& Newcombe, P. A. (2008). Measuring selfefficacy in gambling: the gambling refusal self-efficacy questionnaire. Journal of Gambling Studies, 24, 229-246.

Crisp, B. R., Thomas, S. A., Jackson, A. C., Thomason, N., Smith, S., Borrell, J., Ho, W., \& Holt, T. A. (2000). Sex differences in the treatment needs and outcomes of problem gamblers. Research on Social Work Practice, 10, 229-242.

Diener, E., Emmons, R. A., Larsen, R. J., \& Griffin, S. (1985). The satisfaction with life scale. Journal of Personality Assessment, 49, 71-75.

Ferris, J., \& Wynne, H. J. (2001). The Canadian problem gambling index final report. Ottawa: Canadian Centre on Substance Abuse.

Hodgins, D. C., \& el-Guebaly, N. (2004). Retrospective and prospective reports of precipitants to relapse in pathological gambling. Journal of Consulting \& Clinical Psychology, 72, 72-80.

Hodgins, D. C., Peden, N., \& Makarchuk, K. (2004). Self-efficacy in pathological gambling treatment outcome: development of a gambling abstinence self-efficacy scale (GASS). International Gambling Studies, 4, 99108.

Johansson, A., Grant, J. E., Kim, S. W., Odlaug, B. L., \& Götestam, K. G. (2009). Risk factors for problematic gambling: a critical literature review. Journal of Gambling Studies, 25, 67-92.

Lai, D. W. L. (2006). Gambling and the older Chinese in Canada. Journal of Gambling Studies, 22, 121-141.

Lesieur, H. R., \& Blume, S. B. (1987). The South Oaks Gambling Screen (SOGS): a new instrument for the identification of pathological gamblers. American Journal of Psychiatry, 144, 1184-1188.

Lovibond, S. H., \& Lovibond, P. F. (1995). Manual for the depression anxiety stress scales (2nd ed.). Sydney: Psychology Foundation.

Neal, P., Delfabbro, P. H., \& O’Neil, M. (2005). Problem Gambling and Harm: Towards a National Definition. Melbourne: Report prepared for the National Gambling Research Program Working Party.

Oei, T. P., Lin, J., \& Raylu, N. (2008a). The relationship between gambling cognitions, psychological states, and gambling: a cross-cultural study of Chinese and Caucasians in Australia. Journal of Cross-Cultural Psychology, 39, 147-161. 
Oei, T. P. S., Raylu, N., \& Grace, R. (2008b). Self help program for problem gamblers. Queensland: University of Queensland.

Oei, T. P. S., Raylu, N., \& Casey, L. M. (2010). Effectiveness of group and individual formats of a combined motivational interviewing and cognitive behavioral treatment program for problem gambling: a randomized controlled trial. Behavioural \& Cognitive Psychotherapy, 38, 233-238.

Petry, N. M. (2009). Disordered gambling and its treatment. Cognitive \& Behavioral Practice, 16, $457-467$.

Raylu, N., \& Oei, T. P. S. (2004a). The Gambling Related Cognitions Scale (GRCS): development, confirmatory factor validation and psychometric properties. Addiction, 99, 757-769.

Raylu, N., \& Oei, T. P. S. (2004b). The Gambling Urge Scale (GUS): development, confirmatory factor validation and psychometric properties. Psychology of Addictive Behaviors, 18, 100-105.

Raylu, N., \& Oei, T. P. S. (2010). A cognitive behavioural therapy program for problem gambling: Therapists manual. UK: Routledge.

Rush, B. R., Bassani, D. G., Urbanoski, K. A., \& Castel, S. (2008). Influence of co-occurring mental and substance use disorders on the prevalence of problem gambling in Canada. Addiction, 103, 1847-1856.

Sander, W., \& Peters, A. (2009). Pathological gambling: influence of quality of life and psychological distress on abstinence after cognitive-behavioral inpatient treatment. Journal of Gambling Studies, 25, 253-262.

Saunders, J. B., Aasland, O. G., Babor, T. F., de la Puente, J. R., \& Grant, M. (1993). Development of the Alcohol Use Disorders Screening Test (AUDIT). WHO collaborative project on early detection of persons with harmful alcohol consumption. II. Addiction, 88, 791-804.

Ste-Marie, C., Gupta, R., \& Derevensky, J. L. (2006). Anxiety and social stress related to adolescent gambling behavior and substance use. Journal of Child \& Adolescent Substance Abuse, 15, 55-74.

Toneatto, T., Boughton, R., \& Borsoi, D. (2002). A comparison between male and female gamblers. https:// ozone.scholarsportal.info/bitstream/1873/5893/1/10316422.pdf.

Westphal, J. R., \& Johnson, L. J. (2007). Multiple co-occurring behaviours among gamblers in treatment: implications and assessment. International Gambling Studies, 7, 73-99.

WHOQOL Group. (1998). Development of the World Health Organization WHOQOL-BREF Quality of Life Assessment. Psychological Medicine, 28, 551-558.

Winslow, L. H. (2002). The relationship of gambling on depression, perceived social support, and life satisfaction in an elderly sample. Dissertation Abstracts International: Section B: the Sciences \& Engineering, 62, 4770.

Wong, V. W. K., Chan, E. K. M., Tai, S. P. K., \& Tao, V. Y. K. (2008). Problem gambling among university students in Macao. Journal of Psychology in Chinese Societies. Special Issue: Liberalization of Macao's Casino Industry \& Psychological Phenomena, 9, 47-66. 